\section{Fatores ambientais associados à obesidade em população adulta de um município brasileiro de médio porte}

\author{
Environmental factors associated with obesity \\ in the adult population in a medium-sized \\ Brazilian city
}

\section{Factores ambientales asociados a la obesidad en una población adulta de un municipio brasileño de tamaño medio}

Fernanda Maria Oliveira da Silva 1

Taiane Gonçalves Novaes 1

Andréia Queiroz Ribeiro 1

Giana Zarbato Longo 1

Milene Cristine Pessoa 2

\section{Resumo}

O objetivo deste trabalho foi estimar as associações entre as variáveis ambientais e a obesidade em população adulta de um município brasileiro de médio porte. Trata-se de estudo transversal com dados individuais de estudo de base populacional e dados ambientais obtidos por meio de observação direta, posteriormente georreferenciados com base nos endereços. A amostra incluiu 965 adultos de 20 a 59 anos. Utilizaram-se dados antropométricos, socioeconômicos, demográficos, comportamentais e de percepção de saúde. A variável desfecho foi a obesidade, definida por índice de massa corporal (IMC) $\geq 30 \mathrm{~kg} / \mathrm{m}^{2}$. Os estabelecimentos de venda de alimentos foram classificados em: supermercados, estabelecimentos com predominância de alimentos saudáveis, não saudáveis e mistos. O ambiente para a prática de atividade física foi categorizado em público e privado. Para a caracterização do ambiente social utilizou-se a renda per capita do setor censitário em terços e taxa de criminalidade. Realizou-se análise de regressão logística binária pelo modelo de equações de estimativa generalizadas. Foi verificada associação inversa entre a densidade dos locais públicos e privados para a prática de atividade física e obesidade $(O R=0,95$, IC95\%: 0,92-0,99; OR = 0,98, IC95\%: 0,97-0,99), em modelos ajustados por variáveis individuais e ambientais. Em todos os modelos o terço de renda per capita mais alto associou-se inversamente à obesidade ( $p \leq$ 0,05). O ambiente alimentar e as taxas de criminalidade não foram independentemente associados à obesidade. Tais achados sugerem que a renda do setor censitário e o ambiente de atividade física podem direcionar políticas públicas para a diminuição da obesidade no município.

Obesidade; Meio Ambiente e Saúde Pública; Saúde do Adulto
Correspondência

F. M. O. Silva

QNB 2 lote 13 casa 1, Taguatinga, DF 72115-020, Brasil.

fernandanutufv@yahoo.com.br

1 Universidade Federal de Viçosa, Viçosa, Brasil.

2 Universidade Federal de Minas Gerais, Bolo Horizonte, Brasil. 


\section{Introdução}

A obesidade é uma doença complexa e multifatorial, resultando da interação de genes, fatores ambientais, psicossociais e de estilos de vida 1 . Essa, por sua vez, é um dos principais fatores de risco para outras doenças crônicas 2 , contribuindo para o aumento da morbidade e mortalidade em vários países 3 .

Dados da Organização Mundial da Saúde (OMS) 2 apontam que a prevalência global de obesidade em 2014 foi de 13\% (650 milhões) nos adultos. No Brasil, os dados da Pesquisa Nacional de Saúde ${ }^{4}$ mostraram prevalência de 20,8\% em indivíduos acima de 18 anos.

Nas últimas décadas, o aumento da prevalência de obesidade em países em desenvolvimento ocorreu em virtude de mudanças acarretadas pelos processos de urbanização e transição nutricional 5 . Essas mudanças favoreceram o consumo de produtos ultraprocessados e impactaram nos níveis de atividade física das pessoas, contribuindo para um balanço energético positivo e consequentemente para um maior ganho de peso 6,7.

A complexa etiologia da obesidade demanda elaborada estratégia de saúde pública para o seu enfrentamento, uma vez que somente as características individuais não são capazes de elucidar a expansão de sua prevalência em nível populacional ${ }^{8}$. Nessa perspectiva, na década de 1990 foi proposto um modelo teórico que buscou elucidar a influência do ambiente sobre a obesidade. Segundo os autores, locais que oferecem poucas opções ou pouca diversidade de estabelecimentos de venda de alimentos, ou ainda, oferecem escassas oportunidades e estruturas de prática de atividade física são considerados "ambientes obesogênicos" 9.

Diante disso, tem crescido o interesse em pesquisas abordando a relação entre o ambiente e a obesidade. Estudos prévios realizados em países desenvolvidos 10,11 e no Brasil 12,13, apontam que um ambiente alimentar favorável, caracterizado por maior disponibilidade e acesso a estabelecimentos de vendas de alimentos saudáveis, pode favorecer hábitos alimentares saudáveis e menor prevalência de obesidade 14 .

Outro aspecto mais recente abordado nos estudos é o papel que o acesso e a disponibilidade de locais para a prática de atividade física exerce nos níveis desta atividade e seu possível impacto na moderação do ganho de peso 15,16,17.

Outra vertente investiga ainda a relação entre o ambiente social, que compreende as relações, grupos e processos sociais que existem entre indivíduos de uma determinada vizinhança e sua influência no estado nutricional. Adicionalmente, características de criminalidade do ambiente no entorno das residências também podem impactar no ganho de peso pela falta de segurança para a prática de atividade física, contribuindo para o risco de sobrepeso e obesidade 18,19,20.

Apesar do interesse por estudos que abordam a influência de fatores ambientais sobre a obesidade ter aumentado nos últimos anos no Brasil, ainda existem poucos trabalhos realizados em cidades de médio porte. Além disso, a maioria dos estudos sobre a temática é realizada em países desenvolvidos, os quais têm características socioculturais específicas. Desse modo, compreender o papel dos fatores ambientais associados à obesidade em uma cidade de médio porte é importante para a proposição de estratégias de planejamento urbano, bem como ações específicas para a promoção da saúde e prevenção de doenças.

Frente ao exposto, o objetivo do presente trabalho foi estimar as associações entre as variáveis ambientais e a obesidade em população adulta de um município de médio porte.

\section{Métodos}

Trata-se de um estudo transversal, realizado com indivíduos na faixa etária de 20 a 59 anos, de ambos os sexos, e residentes na zona urbana de Viçosa, Minas Gerais, Brasil. A cidade está localizada na Região da Zona da Mata no Estado de Minas Gerais (Brasil) e conta com uma população de 72.220 habitantes, território de $299 \mathrm{~km}^{2}$, densidade populacional de 241,2 habitantes $/ \mathrm{km}^{2}$, 99 setores censitários na região urbana e 11 na zona rural 21. 


\section{População do estudo e amostragem}

A população adulta de Viçosa na faixa etária estudada, segundo o Censo Demográfico de 2010, era de 43.431 indivíduos 21. O tamanho da amostra foi definido considerando o nível de confiança de 95\%, sendo o cálculo realizado no programa Epi Info (https://www.cdc.gov/epiinfo/index.html), levandose em conta os seguintes parâmetros: população de referência: 43.431; prevalência do desfecho de 20,8\% 4; erro amostral previsto de 3,5\%; efeito do desenho do estudo estimado em 1,5; totalizado 728 indivíduos. A esses, somaram-se $20 \%$ referentes a perdas ou recusas e $10 \%$ para o controle dos fatores de confusão, obtendo-se uma amostra final de 1.011 indivíduos. Ocorreram perdas e recusas (4,5\%), mantendo-se o poder do estudo. Assim, foram efetivamente entrevistados 965 adultos.

Foram consideradas perdas os domicílios visitados, pelo menos quatro vezes, incluída uma visita em finais de semana e outra noturna, sem que o entrevistador localizasse a pessoa a ser entrevistada. No caso de residências desabitadas, dirigiu-se à primeira casa à direita. O motivo da perda foi identificado para aquela residência e/ou indivíduo e anotado na planilha do entrevistador 22 .

Os indivíduos que se recusaram a participar da pesquisa foram novamente contatados pelos supervisores. Aqueles que mantiveram a não concordância para a participação foram denominados recusas. Gestantes, indivíduos acamados ou impossibilitados para a mensuração das medidas e aqueles com alguma deficiência mental que impedisse de responder às questões não eram elegíveis para o estudo 22.

Para a obtenção desta amostra foi realizado um processo de amostragem por conglomerado, em duplo estágio, tomando como unidades, respectivamente, os setores censitários, unidades de recenseamento do Instituto Brasileiro de Geografia e Estatística (IBGE) e os quarteirões 23.

Sortearam-se 30 setores censitários dentre os 99 existentes na zona urbana de Viçosa 23. Cada setor censitário recebeu um número antes do sorteio, que foi realizado por amostragem casual simples, sem reposição, usando-se tabelas de números aleatórios 24. Posteriormente, foi realizado o sorteio dos quarteirões e das esquinas, iniciando-se o trabalho de campo no sentido horário a partir da esquina sorteada.

A população da faixa etária de interesse do estudo corresponde a 52\% da população de Viçosa. Tendo em vista que cada setor censitário tem 300 domicílios e média de quatro moradores em cada residência, considerou-se que havia 1.200 pessoas por setor censitário, sendo 624 na faixa etária contemplada. Dessa forma, como a amostra calculada correspondeu a 1.011 indivíduos, e considerandose 30 setores censitários, estimou-se a necessidade de obtenção de 34 pessoas em cada um dos setores sorteados. Assim, deveriam ser visitados 10 domicílios por setor censitário, para perfazer o número de indivíduos necessários para a pesquisa.

\section{Coleta e calibração de dados}

A etapa de coleta dos dados individuais ocorreu entre junho de 2012 e maio de 2014 valendo-se da pesquisa: Sindrome Metabólica e Fatores Associados: Um Estudo de Base Populacional em Adultos de Viçosa/ $M G$. Os dados ambientais foram obtidos baseando-se na pesquisa Levantamento de Dados do Ambiente Construído da Zona Urbana de Viçosa (MG) entre dezembro de 2015 e julho de 2016.

Os dados individuais foram obtidos por meio da aplicação de questionários, em visitas domiciliares, por duplas de entrevistadores treinados que, seguindo os critérios determinados, convidaram todas as pessoas que atendiam ao critério de inclusão para participarem da pesquisa. Posteriormente, foram coletados dados de peso e altura dos participantes do estudo, nas dependências da Universidade Federal de Viçosa.

Para a coleta dos dados individuais realizou-se um treinamento prévio a respeito dos objetivos do estudo, critérios, métodos utilizados para a mensuração de todas as variáveis e foi realizada uma etapa de calibração de medidas antropométricas. Ao final, foram analisadas as concordâncias obtidas entre os diferentes avaliadores e o avaliador padrão por meio do coeficiente de correlação intraclasse. Foi realizado ainda o pré-teste dos questionários com o objetivo de adequação ao trabalho de campo 23.

A segunda fase da coleta compreendeu o levantamento por observação direta de dados ambientais referentes aos estabelecimentos de vendas de gêneros alimentícios e locais de prática de atividade 
física, por meio de questionários. Para este trabalho foram utilizadas as informações dos estabelecimentos existentes à época da coleta de dados individuais.

Anteriormente à coleta de dados ambientais, realizou-se um estudo piloto na região central do Município de Viçosa, quando foi observada a dinâmica da obtenção de dados e a viabilidade da aplicação dos questionários utilizados.

\section{Caracterização das variáveis do estudo}

Para o presente trabalho a variável dependente foi a obesidade. Foram mensuradas a massa corporal $(\mathrm{kg})$ e estatura, medidas de acordo com Lohman et al. 25 , para o cálculo do índice de massa corporal (IMC). Foram classificados como obesos os indivíduos com IMC $\geq 30 \mathrm{~kg} / \mathrm{m}^{2} 26$.

As variáveis independentes incluíram as variáveis sociodemográficas: idade (em anos), sexo, estado civil (com companheiro e sem companheiro), escolaridade em anos (0-4 anos; 5-8; 9-11; $\geq 12$ anos) 21 e classe socioeconômica avaliada com base na posse de bens de consumo e utilidades domésticas, de acordo com a Associação Brasileira de Empresas de Pesquisa (ABEP) 27. Posteriormente categorizada em alta (classes A1, A2, B1 e B2), intermediária (classes C1 e C2) e baixa (classes D e E).

O bloco de variáveis comportamentais englobou hábitos alimentares, nível de atividade física, comportamento sedentário (tempo de tela) e tabagismo. Os hábitos alimentares foram avaliados tendo como base o questionário Vigilância de Fatores de Risco e Proteção para Doenças Crônicas por Inquérito Telefônico (VIGITEL 2012) 28. A análise dos hábitos alimentares baseou-se em marcadores de alimentação saudável (consumo regular em pelo menos cinco vezes na semana de frutas e/ou hortaliças e feijão), e em marcadores de alimentação não saudável (consumo regular em pelo menos cinco vezes na semana de refrigerantes e/ou suco artificial e consumo de carnes com gordura visível).

O tempo de tela foi avaliado pelo somatório de tempo despendido em atividades como assistir à televisão, utilização de computador, ou ainda, em atividades sentadas e categorizado em até 3 horas/ dia e mais de 3 horas/dia 28.

O nível de atividade física no lazer foi avaliado valendo-se do International Questionnaire on Physical Activity (IPAQ) 29, validado para a população brasileira. Para este estudo foi utilizado o domínio 4 (atividades físicas de recreação, esporte, exercício e lazer) por apresentar associações consistentes com o risco de sobrepeso e obesidade, além de ser o domínio mais usado em outros trabalhos realizados no Brasil. O nível de atividade física foi categorizado em insuficientemente ativo $(<150$ minutos de atividades físicas nos últimos sete dias) e ativo ( $\geq 150$ minutos de atividades físicas nos últimos sete dias $) 30$.

O tabagismo foi avaliado em função do consumo atual e passado de cigarros. Foram considerados fumantes os indivíduos que responderam positivamente à questão “ $\mathrm{O}(\mathrm{a})$ senhor(a) fuma?” e ex-fumantes aqueles que responderam positivamente à questão “O(a) senhor(a) já fumou?”, sendo categorizado em: fumantes, ex-fumantes e não fumantes 28 .

A avaliação do ambiente alimentar foi realizada por observação direta, utilizando-se quatro tipos de questionários: instrumento de avaliação de estabelecimentos de venda de alimentos para o consumo em domicílio; instrumento de avaliação de estabelecimentos de venda de alimentos para o consumo imediato; instrumento de avaliação de feiras livres; e instrumento de avaliação de comércios ambulantes. Esses questionários foram adaptados com base no instrumento desenvolvido para a realidade brasileira 31 .

A coleta de dados dos estabelecimentos de venda de alimentos, incluindo a de vendedores ambulantes e estruturas destinadas à prática de atividade física, foi realizada em todas as ruas dos 99 setores censitários urbanos da cidade entre os horários de 08 e 18 horas, de segunda a sexta-feira. Durante o trajeto, foi identificada a presença e o tipo de estabelecimento destinado à venda de alimentos ou para a prática de atividade física. Uma vez que um dos objetivos do levantamento era identificar os estabelecimentos que funcionavam entre 2012 e 2014, acrescentaram-se as seguintes perguntas aos questionários: “Funciona desde quando neste endereço atual?”. Especificamente no questionário de venda de alimentos, acrescentaram-se as perguntas: "Já exerceu outro tipo de atividade? Se sim, qual atividade?". "Sempre vendeu esses produtos? Se não, quais produtos eram vendidos?".

Para a avaliação da relação entre o ambiente alimentar, ambiente para a prática de atividade física e a obesidade em adultos utilizaram-se os dados dos estabelecimentos que funcionavam entre o período de 2012 e 2014. 
Os estabelecimentos de venda de alimentos foram posteriormente agregados em quatro categorias: supermercados; estabelecimentos com predominância de alimentos saudáveis (sacolões, açougues e peixarias, estabelecimentos de venda de laticínios e frios, lojas de produtos naturais, ambulantes de alimentos saudáveis, feiras livres); não saudáveis (lojas de conveniência, mercearias, bares, bombonières, distribuidoras de bebidas, lanchonetes, sorveterias e ambulantes de alimentos não saudáveis); e mistos (restaurantes e padarias) de acordo com proposta de estudo prévio 32 . A categoria de supermercados foi analisada separadamente, em função da ausência de consenso na literatura a respeito da influência do supermercado nas escolhas de compras dos indivíduos, uma vez que são locais que oferecem, além de alimentos saudáveis, várias opções de alimentos industrializados 33. As variáveis do ambiente alimentar foram analisadas por meio da densidade (número do tipo de estabelecimento dividido pela área do setor censitário em $\mathrm{km}^{2}$ ).

Para a avaliação das estruturas públicas e privadas para a prática de atividade física no lazer foi utilizado o instrumento - Physical Activity Resource Assessment (PARA) - adaptado à realidade brasileira 34. As informações incluíram dados sobre todos os estabelecimentos para prática de atividade física no lazer, tanto públicos (praças e academias ao ar livre, além de locais de caminhada - campus universitário) quanto privados (clubes e academias). A análise dos locais para prática de atividade física também foi feita por meio da medida de densidade.

O endereço dos domicílios dos indivíduos, estabelecimentos de vendas de alimentos e locais para a prática de atividade física foram georreferenciados e a densidade de estabelecimentos foi obtida em relação à amostra de obesos e não obesos contemplados na área do setor censitário.

Para a caracterização do ambiente social foram coletados dados sobre criminalidade referentes ao período de 2012 a 2014, obtidos junto à Secretaria de Estado de Defesa Social de Minas Gerais (Secretaria do Estado de Defesa Social de Minas Gerais. Dados de criminalidade: 2017. http://www. numeros.mg.gov.br/QvAJAXZfc/opendoc.htm?document=MapaResultados.qvw\&host=QVS\%40vm13532\&anonymous=true, acessado em Abr/2017). Foram obtidos ainda dados de área, população e renda média mensal dos setores censitários 6. Posteriormente, calculou-se a renda per capita do setor censitário (razão entre o total do rendimento nominal mensal dos domicílios particulares permanentes do setor censitário e a população residente em domicílios particulares permanentes) categorizada em terços 6 e a taxa de criminalidade (total de homicídios, estupros, roubos, sequestro, furtos/mil habitantes).

A variável densidade populacional foi calculada pela razão entre o número de pessoas residentes e a área total em cada um dos setores censitários.

\section{Variáveis de ajuste}

Foram selecionadas como variáveis individuais e ambientais de ajuste aquelas que apresentaram valor de $\mathrm{p}<0,05$ na análise bivariada. Dentre elas, as variáveis individuais: idade, tabagismo, tempo de tela e autorrelato do estado de saúde; e as variáveis ambientais do ambiente social (renda per capita e taxa de criminalidade). A variável sexo foi acrescentada no ajuste devido à sua relevância para a compreensão do desfecho.

\section{Análise estatística}

A análise descritiva dos dados ambientais foi realizada com base em distribuições de frequência, medidas de tendência central e de dispersão. A associação entre as variáveis ambientais e a obesidade foi estimada por meio de regressão logística binária pelo modelo de equações de estimativa generalizadas (GEE, do inglês generalized estimation equations), que considera o efeito agregado dos indivíduos. Foi utilizada a estrutura de correlação exchangeable, recomendada quando as observações estão agrupadas em alguma estrutura específica 35,36 .

Foram construídos quatro modelos distintos para cada categoria de estabelecimento do ambiente alimentar, ajustados pelas variáveis individuais e pela variável ambiental renda per capita do setor censitário categorizada em terços. Para o ambiente de atividade física foi construído um modelo incluindo estabelecimentos públicos e privados, com ajuste por variáveis individuais e ambientais (renda per capita e taxa de criminalidade). 
Os modelos foram selecionados com base em modelo teórico proposto em um estudo internacional 37 , que foi adaptado à realidade brasileira. Dentre outros aspectos considerou-se a relevância das variáveis para o desfecho (teste de Wald), verificação de colinearidade entre as variáveis e valor de QIC (quasilikelihood under the independence model criterion), método específico da regressão GEE para seleção dos melhores modelos.

Foram estimados valores de odds ratio (OR) e seu respectivo intervalo de 95\% de confiança (IC95\%). As análises de dados foram feitas usando-se o software estatístico Stata, versão 13.1 (https://www. stata.com). O nível de significância adotado em todas as análises foi de $5 \%$.

\section{Resultados}

A amostra foi composta por 965 adultos com média de idades de 34,2 anos $( \pm 12,0)$, predominância do sexo feminino $(55,2 \%)$ e prevalência de obesidade de $13,8 \%$.

$\mathrm{Na}$ Tabela 1, encontra-se a descrição das variáveis do ambiente dos setores censitários urbanos de Viçosa. As medianas das densidades de estabelecimentos saudáveis, não saudáveis, mistos e supermercados foram, respectivamente, 0, 2, 0 e 0. Em relação aos locais para a prática de atividade física, as medianas de locais públicos e privados foram, respectivamente, 0 e 1.

A Tabela 2 mostra a distribuição das variáveis ambientais, razão de chances não ajustada e seus IC95\% da regressão logística binária para a obesidade com base no GEE.

$\mathrm{Na}$ análise múltipla (Tabela 3), verificou-se associação inversa entre a densidade dos locais públicos e privados para a prática de atividade física (OR =0,95, IC95\%: 0,92-0,99; OR =0,98, IC95\%: 0,97-0,99) e obesidade mesmo após ajuste. Nos modelos 1 a 5, o terço mais alto de renda per capita associou-se inversamente à obesidade ( $\mathrm{OR}=0,39$, IC95\%: 0,23-0,70; OR = 0,41, IC95\%: 0,25-0,70; $\mathrm{OR}=0,42$, IC95\%: 0,26-0,70; OR = 0,43, IC95\%: 0,30-0,72, respectivamente). A densidade de estabelecimentos saudáveis, não saudáveis, mistos e supermercados e a taxa de criminalidade não foram independentemente associadas à obesidade após ajuste.

Tabela 1

Descrição das variáveis ambientais dos setores censitários de Viçosa, Minas Gerais, Brasil, 2012-2014.

\begin{tabular}{|c|c|c|c|c|}
\hline Variáveis & Mediana & Mínimo & Máximo & $\begin{array}{c}\text { Total de } \\
\text { estabelecimentos }\end{array}$ \\
\hline \multicolumn{5}{|c|}{ Estabelecimentos de venda de alimentos } \\
\hline Saudáveis & 0,00 & 0 & 4 & 57 \\
\hline Não saudáveis & 2,00 & 0 & 12 & 293 \\
\hline Mistos & 0,00 & 0 & 4 & 57 \\
\hline Supermercados & 0,00 & 0 & 1 & 18 \\
\hline \multicolumn{5}{|c|}{ Estabelecimentos para a prática de atividade física } \\
\hline Públicos & 0,00 & 0 & 1 & 41 \\
\hline \multirow[t]{2}{*}{ Privados } & 0,00 & 1 & 3 & 22 \\
\hline & Média (DP) & Mínimo & Máximo & $\begin{array}{c}\text { Total de } \\
\text { estabelecimentos }\end{array}$ \\
\hline Renda per capita & $1.070,91(743,99)$ & 219,72 & $2.994,32$ & Não se aplica \\
\hline Taxas de criminalidade & $38,97(60,01)$ & 1,83 & 323,69 & Não se aplica \\
\hline
\end{tabular}

DP: desvio padrão. 


\section{Tabela 2}

Distribuição das variáveis do ambiente alimentar, locais para a prática de atividade física e ambiente social, de acordo com a obesidade em adultos de Viçosa, Minas Gerais, Brasil, 2012-2014.

\begin{tabular}{|c|c|c|c|c|c|}
\hline Variáveis ambientais & $\begin{array}{c}\text { Total } \\
(\mathrm{N}=965) \\
\text { Mediana (IIQ) }\end{array}$ & $\begin{array}{l}\text { Não obesos } \\
\quad(n=832) \\
\text { Mediana (IIQ) }\end{array}$ & $\begin{array}{c}\text { Obesos } \\
(n=133) \\
\text { Mediana (IIQ) }\end{array}$ & $\begin{array}{l}\text { OR não } \\
\text { ajustada }\end{array}$ & IC95\% \\
\hline \multicolumn{6}{|c|}{$\begin{array}{l}\text { Densidade de estabelecimentos de venda } \\
\text { de alimentos }\end{array}$} \\
\hline Saudáveis (número/km²) & $0,00(0,00-0,00)$ & $0,00(0,00-0,32)$ & $0,00(0,00-5,43)$ & 1,00 & $0,99-1,02$ \\
\hline Não saudáveis (número/km²) & $15,90(12,68-15,90)$ & $15,90(12,68-15,90)$ & $12,68(11,53-20,80)$ & 1,00 & $0,99-1,00$ \\
\hline Mistos (número/km²) & $0,00(0,00-0,00)$ & $0,00(0,00-0,00)$ & $0,00(0,00-0,00)$ & 1,00 & $0,99-1,02$ \\
\hline Supermercados (número/km²) & $0,00(0,00-0,00)$ & $0,00(0,00-0,00)$ & $0,00(0,00-0,00)$ & 0,98 & $0,94-1,00$ \\
\hline \multicolumn{6}{|c|}{$\begin{array}{l}\text { Densidade de locais para a prática de } \\
\text { atividade física }\end{array}$} \\
\hline Públicos (número/km²) & $0,00(0,00-0,00)$ & $0,00(0,00-0,00)$ & $0,00(0,00-0,00)$ & 0,93 & $0,86-1,00$ \\
\hline Privados (número/km²) & $0,00(0,00-0,00)$ & $1,40(0-1,68)$ & $0,00(0,00-0,00)$ & 0,95 & $0,92-0,98$ \\
\hline Taxa de criminalidade & $17,13(17,13-18,44)$ & $18,44(17,13-21,42)$ & $14,85(13,35-16,20)$ & 0,98 & $0,98-0,99$ \\
\hline \multirow[t]{2}{*}{ Densidade populacional (km²) } & $\begin{array}{c}5.710,79 \\
(5.576,2-5.786,34)\end{array}$ & $\begin{array}{c}5.710,27 \\
(5.710,27-5.940,79)\end{array}$ & $\begin{array}{c}4.800,92 \\
(3.948,92-6.332,79)\end{array}$ & 0,99 & $0,99-1,00$ \\
\hline & n (\%) & n (\%) & n (\%) & $\begin{array}{l}\text { OR não } \\
\text { ajustada }\end{array}$ & IC95\% \\
\hline \multicolumn{6}{|l|}{ Renda per capita (terços) } \\
\hline 1으 & $183(19,86)$ & $146(17,55)$ & $37(27,82)$ & Ref. & \\
\hline 2o & $412(42,69)$ & $343(41,23)$ & $69(51,88)$ & 0,82 & $0,52-1,30$ \\
\hline 3o & $370(38,34)$ & $343(41,23)$ & $27(20,30)$ & 0,41 & $0,23-0,72$ \\
\hline
\end{tabular}

DP: desvio padrão; IC95\%: intervalo de 95\% de confiança; IIQ: intervalo interquartílico; OR: odds ratio; Ref.: referência.

\section{Discussão}

A prevalência de obesidade entre adultos de Viçosa é de 13,8\%, e estudos de abrangência nacional 4,38 apontam prevalências globais de 18,9\% e 20,8\% na população brasileira. A média de idades da amostra revela uma população adulta jovem, o que pode explicar, ao menos em parte, essas menores prevalências de obesidade. Apesar de baixa se comparada às prevalências nacionais, ainda é considerável, tendo em vista os níveis crescentes de excesso de peso observados no Brasil.

Os resultados deste estudo indicaram que uma maior densidade de estabelecimentos públicos e privados para a prática de atividade física e o terço mais alto de renda per capita do setor censitário associaram-se independentemente a menores chances de obesidade nos adultos.

Cabe destacar que o ambiente para a prática de atividade física na cidade é caracterizado pela predominância de estabelecimentos privados em relação aos públicos, localizados principalmente na região central. Adicionalmente, nas regiões periféricas, verificam-se extensas áreas em que a presença de locais públicos e privados para a prática de atividade física é escassa. Características semelhantes às observadas em capitais brasileiras onde foram realizados estudos sobre ambiente e excesso de peso, como São Paulo e Belo Horizonte 13,39.

Além disso, apesar de não ter sido analisado o possível motivo para a utilização dos locais de prática de atividade física, cabe ressaltar que a cidade conta com um campus universitário localizado em área central, semelhante a parques existentes em grandes cidades e com espaço para caminhadas e atividades ao ar livre. Dessa forma, por se tratar de uma cidade de médio porte, muitas pessoas se deslocam de outros bairros para a realização de atividades físicas no local, o que pode contribuir para menores prevalências de obesidade no município. 
Tabela 3

Modelos ajustados para a obesidade de acordo com as variáveis individuais e ambientais em adultos de Viçosa, Minas Gerais. Brasil, $2012-2014$.

\begin{tabular}{|c|c|c|c|c|c|c|c|c|c|c|}
\hline \multirow[t]{2}{*}{ Variáveis } & \multicolumn{2}{|c|}{ Modelo 1} & \multicolumn{2}{|c|}{ Modelo 2} & \multicolumn{2}{|c|}{ Modelo 3} & \multicolumn{2}{|c|}{ Modelo 4} & \multicolumn{2}{|c|}{ Modelo 5} \\
\hline & $\begin{array}{c}\text { OR } \\
\text { ajustada }\end{array}$ & IC95\% & $\begin{array}{c}\text { OR } \\
\text { ajustada }\end{array}$ & IC95\% & $\begin{array}{c}\text { OR } \\
\text { ajustada }\end{array}$ & IC95\% & $\begin{array}{c}\text { OR } \\
\text { ajustada }\end{array}$ & IC95\% & $\begin{array}{c}\text { OR } \\
\text { ajustada }\end{array}$ & IC95\% \\
\hline \multicolumn{11}{|l|}{ Individuais } \\
\hline \multicolumn{11}{|l|}{ Sexo } \\
\hline Feminino & 1,00 & - & 1,00 & - & 1,00 & - & 1,00 & - & 1,00 & - \\
\hline Masculino & 1,17 & $0,81-1,70$ & 1,18 & $0,82-1,71$ & 1,19 & $0,78-1,80$ & 1,18 & $0,82-1,71$ & 1,18 & $0,83-1,68$ \\
\hline Idade & 1,03 & $1,01-1,05$ & 1,03 & $1,01-1,05$ & 1,03 & $1,01-1,04$ & 1,03 & $1,01-1,04$ & 1,03 & $1,01-1,04$ \\
\hline \multicolumn{11}{|l|}{ Tabagismo } \\
\hline \multicolumn{11}{|l|}{ Não fumante } \\
\hline Fumante & 0,70 & $0,40-1,22$ & 0,69 & $0,40-1,20$ & 0,69 & $0,36-134$ & 0,7 & $0,40-1,21$ & 0,69 & $0,40-1,17$ \\
\hline Ex-fumante & 2,07 & $1,28-3,34$ & 2,10 & $1,31-3,35$ & 2,04 & $1,26-3,30$ & 2,08 & $1,28-3,35$ & 2,03 & $1,26-3,26$ \\
\hline \multicolumn{11}{|l|}{ Tempo de tela (horas/dia) } \\
\hline \multicolumn{11}{|l|}{$<3$} \\
\hline$\geq 3$ & 1,78 & $1,24-2,56$ & 1,80 & $1,25-2,61$ & 1,78 & $1,18-2,65$ & 1,79 & $1,24-2,57$ & 1,75 & $1,23-2,49$ \\
\hline \multicolumn{11}{|l|}{ Autorrelato de saúde } \\
\hline \multicolumn{11}{|l|}{ Regular/Ruim } \\
\hline \multicolumn{11}{|l|}{ Excelente } \\
\hline \multicolumn{11}{|l|}{ Ambientais } \\
\hline \multicolumn{11}{|l|}{$\begin{array}{l}\text { Densidade de } \\
\text { estabelecimentos }\end{array}$} \\
\hline Saudáveis & 1,00 & $0,99-1,01$ & - & - & - & - & - & - & - & - \\
\hline Não saudáveis & - & - & 0,99 & $0,99-1,00$ & - & - & - & - & - & - \\
\hline Mistos & - & - & - & - & 1,00 & $0,98-1,01$ & - & - & - & - \\
\hline Supermercados & - & - & - & - & - & - & 0,99 & $0,96-1,02$ & - & - \\
\hline \multicolumn{11}{|c|}{$\begin{array}{l}\text { Densidade de locais para a } \\
\text { prática de atividade física }\end{array}$} \\
\hline Públicos & - & - & - & - & - & - & - & - & 0,95 & $0,92-0,99$ \\
\hline Privados & - & - & - & - & - & - & - & - & 0,98 & $0,97-0,99$ \\
\hline \multicolumn{11}{|l|}{ Renda per capita (terços) } \\
\hline 10 & 1,00 & - & 1,00 & - & 1,00 & - & 1,00 & - & 1,00 & - \\
\hline 2o & 0,83 & $0,59-1,16$ & 0,90 & $0,55-1,46$ & 0,84 & $0,56-1,24$ & 0,87 & $0,64-1,19$ & 0,93 & $0,68-1,27$ \\
\hline 3으 & 0,41 & $0,25-0,67$ & 0,45 & $0,25-0,81$ & 0,43 & $0,27-0,66$ & 0,44 & $0,28-0,70$ & 0,59 & $0,38-0,93$ \\
\hline Taxas de criminalidade & - & - & - & - & - & - & - & - & 0,99 & $0,99-1,00$ \\
\hline
\end{tabular}

IC95\%: intervalo de 95\% de confiança; OR: odds ratio.

Nota: Modelos 1 a 4: ajustados por variáveis individuais e variável ambiental renda per capita do setor censitário em terços; Modelo 5: ajustado por variáveis individuais e variáveis ambientais (renda per capita do setor censitário em terços e taxas de criminalidade).

Jaime et al. 13, ao avaliarem as características do ambiente construído e sua relação com sobrepeso em São Paulo, encontraram correlação positiva de maior densidade de parques e locais públicos para a prática de atividade física e menores prevalências de sobrepeso, ajustando para o índice de desenvolvimento humano (IDH). Assim como os achados de dois outros trabalhos realizados em Belo Horizonte, em que o aumento do número de locais para a prática de atividade física (públicos e privados) foi associado com menores chances de obesidade, em modelos ajustados por sexo, idade, nível educacional e consumo de carne com gordura visível 14,40. Resultados semelhantes aos encontrados no presente estudo, em modelos ajustados por variáveis individuais e ambientais semelhantes. 
Estudos internacionais realizados nos Estados Unidos com amostras representativas apontaram resultados similares 41,42 . Entretanto, um trabalho realizado na Austrália 43 não encontrou associação entre a disponibilidade de locais destinados à prática de atividade física e o IMC em adultos, usando fontes ambientais secundárias. Os autores justificam que além da disponibilidade, fatores comportamentais e relacionados à infraestrutura desses locais também podem influenciar em sua utilização.

Aspectos da vizinhança como maior distância e tempo de deslocamento, menor disponibilidade de locais para a prática de atividades e infraestrutura inadequada podem dificultar a adesão à prática de atividades físicas. Dessa forma, a proximidade física pode colaborar para a redução de possíveis barreiras psicológicas e ambientais associadas à prática de atividade física, bem como contribuir em parte com a diminuição da obesidade 44,45 .

Os achados deste trabalho confirmam os de estudos prévios, apesar disto, cabe destacar que somente a presença de locais de prática de atividade física não implica sua utilização, uma vez que estruturas precárias e falta de incentivo são fatores que podem constituir barreiras 46. Dessa forma, as políticas para a diminuição da prevalência de obesidade no município devem ser direcionadas ao aumento de estruturas públicas para atividade física em conjunto com a implantação de programas para estas atividades.

Associações de variáveis do ambiente social como renda per capita do setor censitário também foram testadas neste estudo. O terço mais alto de renda média per capita associou-se inversamente à obesidade em todos os modelos. Os resultados de estudos nacionais e internacionais mostraram resultados semelhantes 14,42,47, constatando que vizinhanças de maior renda frequentemente apresentam menores prevalências de obesidade.

Pesquisas têm demonstrado que a renda da vizinhança influencia tanto características individuais quanto relacionadas ao ambiente do entorno. No nível ambiental, regiões mais favorecidas economicamente podem propiciar maior acesso e disponibilidade de estabelecimentos de vendas de alimentos, com preços mais acessíveis, além de maior disponibilidade e proximidade de locais para a prática de atividade física, com melhores infraestruturas 42,48 . O nível individual de maior renda pode favorecer maior autonomia em relação aos hábitos relacionados à alimentação e prática de atividade física e contribuir para melhores prognósticos em saúde, principalmente por favorecer maior acesso a serviços de saúde 31,49 .

Um estudo prévio realizado na cidade constatou que setores censitários em maiores terços de renda apresentavam maior número de estabelecimentos saudáveis e mistos e menor número de estabelecimentos não saudáveis. Já em setores censitários periféricos e de baixa renda verificou-se baixa disponibilidade de estabelecimentos saudáveis e aglomerações de estabelecimentos não saudáveis 50 . Tal fato pode propiciar um ambiente obesogênico, principalmente em regiões periféricas, configurando-se como possíveis "desertos alimentares" 50.

Essa realidade é semelhante à encontrada em países desenvolvidos, nos quais geralmente a densidade de estabelecimentos considerados não saudáveis tende a ser maior em regiões desfavorecidas economicamente 51,52 . No entanto, em países em desenvolvimento o fator renda pode ser ainda mais crucial, uma vez que em regiões periféricas as pessoas têm menos acesso a serviços de transporte público, enfrentam maiores distâncias e frequentemente baseiam suas escolhas alimentares em função do preço 52 .

Ainda no contexto do ambiente social, as taxas de criminalidade não foram associadas à obesidade, assim como em outros estudos realizados no Brasil 13,17,39. A hipótese seria a de que maiores taxas de criminalidade da vizinhança podem constituir barreiras para a prática de atividade física, desencorajando práticas ao ar livre e no período noturno. O estudo de Mendes et al. 53 encontrou associação direta entre taxas de homicídio e prevalência de obesidade em Belo Horizonte, utilizando medida de criminalidade diferente da adotada neste trabalho. Apesar disso, para cidades de menor porte, a criminalidade parece não ter o mesmo impacto em relação à obesidade do que em cidades de porte maior. Adicionalmente, ressalta-se a possibilidade de sub-registros de ocorrências de crimes.

Apesar de o ambiente alimentar na cidade ser potencialmente obesogênico, com a presença de pântanos alimentares (áreas com maior disponibilidade de alimentos não saudáveis, ultraprocessados e de baixa qualidade nutricional) 54 e praticamente indisponibilidade de estabelecimentos de venda de alimentos in natura e minimamente processados em áreas periféricas, não foram verificadas associações entre a densidade dos tipos de estabelecimentos de venda de alimentos e a obesidade. 
Sabe-se que além de informações sobre o ambiente alimentar comunitário, que foi o foco do presente estudo, é importante considerar outras dimensões do ambiente alimentar e a dinâmica de relações entre os diferentes tipos de estabelecimentos, para que se tenha uma compreensão completa sobre as relações do mesmo com desfechos como a obesidade 55 .

Nas cidades de médio porte, a distribuição dos estabelecimentos pode ocorrer de forma diferente em relação aos grandes centros urbanos, além disto, frequentemente os indivíduos deslocam-se de seu bairro de origem para a região central, atraídos por supermercados com preços mais acessíveis e disponibilidade de feiras de alimentos, o que pode influenciar em suas escolhas alimentares.

Buscando explorar algumas dessas relações, testamos diferentes modelos utilizando combinações das categorias dos estabelecimentos de alimentos e usando a densidade (medida de exposição ao ambiente alimentar) calculada tanto por área quanto pela população, não sendo encontrados resultados distintos dos apresentados. Além disso, a categoria de supermercado foi incorporada aos modelos tanto de forma isolada quanto englobada à categoria de estabelecimentos mistos. Em ambas as formas não houve associação com a obesidade.

Este trabalho apresenta algumas limitações, uma delas é o fato de não terem sido realizadas auditorias a respeito de informações do microambiente alimentar, como variedade, disponibilidade, preço e qualidade dos alimentos disponibilizados. Outra limitação refere-se a uma possível subestimação do número dos estabelecimentos de venda de alimentos que tenham fechado antes do início da pesquisa. No entanto, não houve subestimação em relação aos estabelecimentos que não têm registro (vendedores informais).

Além disso, foram avaliados apenas dados relacionados ao entorno do domicílio, no entanto, uma abordagem mais completa inclui a análise do ambiente alimentar do entorno do local de trabalho/ estudo, bem como análise mais específica do ambiente domiciliar.

A despeito das limitações, ressalta-se a qualidade tanto dos dados individuais quanto dos ambientais, obtidos de forma direta, o que fortalece os achados. São escassos os estudos em municípios de médio porte e os resultados encontrados neste trabalho são semelhantes aos registrados em pesquisas internacionais e em cidades brasileiras de grande porte.

\section{Conclusão}

Os achados deste estudo sugerem que a renda per capita do setor censitário e a disponibilidade de locais públicos e privados para a prática de atividade física, associadas a características individuais como idade, tempo gasto em atividades sedentárias e tabagismo, devem ser consideradas no planejamento das ações para a prevenção e o controle da obesidade no município. 


\section{Colaboradores}

F. M. O. Silva participou do delineamento e concepção do estudo, análise estatística e interpretação dos dados, redação do artigo e revisão crítica do conteúdo. T. G. Novaes colaborou no delineamento e concepção do estudo, análise estatística dos dados e revisão crítica do conteúdo. A. Q. Ribeiro e G. Z. Longo participaram do delineamento e concepção do estudo e revisão crítica do artigo. M. C. Pessoa contribuiu no delineamento e concepção do estudo, interpretação dos dados, redação e revisão crítica do artigo.

\section{Informações adicionais}

ORCID: Fernanda Maria Oliveira da Silva (00000002-8256-2861); Taiane Gonçalves Novaes (00000002-9180-5490); Andréia Queiroz Ribeiro (00000001-6546-1252); Giana Zarbato Longo (00000001-7666-5007); Milene Cristine Pessoa (00000002-1053-5450).

\section{Agradecimentos}

Os autores agradecem aos adultos participantes, aos alunos participantes, aos alunos de graduação em nutrição e educação física que participaram da coleta de dados ambientais, ao Conselho Nacional de Desenvolvimento Científico e Tecnológico (CNPq) e à Coordenação de Aperfeiçoamento de Pessoal de Nível Superior (Capes) por concessão de bolsa de estudos.

\section{Referências}

1. Schmidhauser S, Eichler K, Brügger U. Environmental determinants of overweight and obesity: extended international literature review. Winterthur: Winterthur Institute of Health Economics; 2009.

2. World Health Organization. Obesity and overweight. http://www.who.int/mediacentre/ factsheets/fs311/en/ (acessado em Mar/2017).

3. Global Burden of Disease Health Financing Collaborator Network. Evolution and patterns of global health financing 1995-2014: development assistance for health, and government, prepaid private, and out-of-pocket health spending in 184 countries. Lancet 2017; 389:1981-2004.

4. Instituto Brasileiro de Geografia e Estatística. Pesquisa Nacional de Saúde 2013 - ciclos de vida: Brasil e grandes regiões. Rio de Janeiro: Instituto Brasileiro de Geografia e Estatística; 2015.

5. Batista Filho M, Rissin A. A transição nutricional no Brasil: tendências regionais e temporais. Cad Saúde Pública 2003; 19 Suppl 1:S181-91.

6. Instituto Brasileiro de Geografia e Estatística. Pesquisa de Orçamentos Familiares 20082009: antropometria e estado nutricional de crianças, adolescentes e adultos no Brasil. Rio de Janeiro: Instituto Brasileiro de Geografia e Estatística; 2010.

7. Organização Pan-Americana da Saúde. Estratégia regional e plano de ação para um enfoque integrado da prevenção e controle de doenças crônicas. Washington DC: Organização Pan-Americana da Saúde; 2007.

8. Michael YL, Nagel CL, Gold R, Hillier TA. Does change in the neighborhood environment prevent obesity in older women? Soc Sci Med 2014; 102:129-37.

9. Swinburn B, Egger G, Raza F. Dissecting obesogenic environments: the development and application of a framework for identifying and prioritizing environmental interventions for obesity. Prev Med 1999; 29:563-70.

10. Hutchinson PL, Nicholas Bodor J, Swalm CM, Rice JC, Rose D. Neighbourhood food environments and obesity in southeast Louisiana. Health Place 2012; 18:854-60.

11. Dubowitz T, Ghosh-Dastidar M, Eibner C, Slaughter ME, Fernandes M, Whitsel EA, et al. The Women's Health Initiative: the food environment, neighborhood socioeconomic status, BMI, and blood pressure. Obesity (Silver Spring) 2012; 20:862-71.

12. Pessoa MC, Mendes LL, Gomes CS, Martins PA, Velásquez-Meléndez G. Food environment and fruit and vegetable intake in a urban population: a multilevel analysis. BMC Public Health 2015; 15:1012.

13. Jaime PC, Duran AC, Sarti FM, Lock K. Investigating environmental determinants of diet, physical activity, and overweight among adults in São Paulo, Brazil. J Urban Health 2011; 88:567-81. 
14. Matozinhos FP, Gomes CS, Andrade ACDS, Mendes LL, Pessoa MC, Friche AA, et al. Neighbourhood environments and obesity among adults: a multilevel analysis of an urban Brazilian context. Prev Med Rep 2015; 2:33741.

15. Morgan Hughey S, Kaczynski AT, Child S, Moore JB, Porter D, Hibbert J. Green and lean: is neighborhood park and playground availability associated with youth obesity? Variations by gender, socioeconomic status, and race/ethnicity. Prev Med 2017; 95 Suppl:S101-8.

16. Jilcott Pitts SB, Edwards MB, Moore JB, Shores KA, DuBose KD, McGranahan D. Obesity is inversely associated with natural amenities and and recreation facilities per capita. J Phys Act Health 2013; 10:1032-8.

17. Gomes CS, Matozinhos FP, Mendes LL, Pessoa MC, Velásquez-Meléndez G. Physical and social environment are associated to leisure time physical activity in adults of a Brazilian City: a cross-sectional study. PLoS One 2016; 11:e0150017.

18. Bennett GG, McNeill LH, Wolin KY, Duncan DT, Puleo E, Emmons KM. Safe to walk? Neighborhood safety and physical activity among public housing residents. PLoS Med 2007; 4:1599-606

19. Hill JL, Chau C, Luebbering CR, Kolivras KK, Zoellner J. Does availability of physical activity and food outlets differ by race and income? Findings from an enumeration study in a health disparate region. Int J Behav Nutr Phys Act 2012; 9:105.

20. Li K, Wen M, Henry K. Residential racial composition and black-white obesity risks: differential effects of neighborhood social and built environment. Int J Environ Res Public Health 2014; 11:626-42.

21. Instituto Brasileiro de Geografia e Estatística. Orçamento e gestão: contagem populacional. Rio de Janeiro: Instituto Brasileiro de Geografia e Estatística; 2011.

22. Segheto W, Silva DCG, Coelho FA, Guimarães RV, Morais SHO, Marins JCB, et al. Body adiposity index and associated factors in adults: method and logistics of a population-based study. Nutr Hosp 2015; 32:101-9.

23. Barros FC, Victora CG. Epidemiologia da saúde infantil: um manual para diagnósticos comunitários. São Paulo: Editora Hucitec/Fundo das Nações Unidas para a Infância; 1998.

24. Silva NN. Amostragem probabilística: um curso introdutório. São Paulo: Edusp; 1998.

25. Lohman TG, Roche AF, Martorell R. Anthropometric standardization reference manual. Champaign: Human Kinetics; 1998.

26. World Health Organization Obesity: preventing and managing the global epidemic. Geneva: World Health Organization; 1998.

27. Associação Brasileira de Empresas de Pesquisa. Critérios de classificação econômica Brasil. São Paulo: Associação Brasileira de Empresas de Pesquisa; 2012.
28. Departamento de Vigilância de Doenças e Agravos Não Transmissíveis e Promoção da Saúde, Secretaria de Vigilância em Saúde, Ministério da Saúde. VIGITEL Brasil 2011: Vigilância de Fatores de Risco e Proteção para Doenças Crônicas por Inquérito Telefônico. Brasília: Ministério da Saúde; 2012.

29. Pardini R, Matsudo SMM, Araújo TL. Validação do questionário internacional de nível de atividade física (IPAQ - versão 6): estudo piloto em adultos jovens brasileiros. Rev Bras Ciênc Mov 2001; 9:45-51.

30. Haskell WL, Lee I, Pate RR, Powell KE, Blair SN. Physical activity and public health: updated recommendation for adults from the American College of Sports Medicine and the American Heart Association. Med Sci Sport Exerc 2007; 39:1423-34.

31. Duran AC. Ambiente alimentar urbano em São Paulo, Brasil: avaliação, desigualdades e associação com consumo alimentar [Tese de Doutorado]. São Paulo: Faculdade de Saúde Pública, Universidade de São Paulo; 2013.

32. Assis MM. Ambiente alimentar residencial e obesidade em crianças e adolescentes de uma cidade de médio porte brasileira [Dissertação de Mestrado]. Juiz de Fora: Faculdade de Medicina, Universidade Federal de Juiz de Fora; 2017.

33. Larsen K, Cook B, Stone MR, Faulkner GEJ. Food access and children's BMI in Toronto, Ontario: assessing how the food environment relates to overweight and obesity. Int J Public Health 2014; 60:69-77.

34. Lee RE, Booth KM, Reese-Smith JY, Regan G, Howard HH. The Physical Activity Resource Assessment (PARA) instrument: evaluating features, amenities and incivilities of physical activity resources in urban neighborhoods. Int J Behav Nutr Phys Act 2005; 2:13.

35. Hanley JA, Negassa A, Edwardes MD, Forrester JE. Statistical analysis of correlated data using generalized estimating equations: an orientation. Am J Epidemiol 2014; 157:364-5.

36. Agranonik M. Equações de Estimação Generalizadas (GEE): aplicação em estudo sobre mortalidade neonatal em gemelares de Porto Alegre - RS (1995-2007) [Dissertação de Mestrado]. Porto Alegre: Universidade Federal do Rio Grande do Sul; 2009.

37. Suglia SF, Shelton RC, Hsiao A, Wang YC, Rundle A, Link BG. Why the neighborhood social environment is critical in obesity prevention. J Urban Health 2016; 93:206-12.

38. Departamento de Vigilância de Doenças e Agravos Não Transmissíveis e Promoção da Saúde, Secretaria de Vigilância em Saúde, Ministério da Saúde. VIGITEL Brasil 2016: Vigilância de Fatores de Risco e Proteção para Doenças Crônicas por Inquérito Telefônico. Brasília: Ministério da Saúde; 2017. 
39. Matozinhos FP, Gomes CS, Costa MA, Mendes LL, Pessoa MC, Velásquez-Meléndez G. Distribuição espacial da obesidade em área urbana no Brasil. Spatial distribution of obesity in an urban Brazilian area. Ciênc Saúde Colet 2015; 20:2779-87.

40. Velásquez-Meléndez G, Mendes LL, Proença Padez CM. Built environment and social environment: associations with overweight and obesity in a sample of Brazilian adults. Cad Saúde Pública 2013; 29:1988-96.

41. Prince SA, Kristjansson EA, Russell K, Billette J, Sawada M, Ali A. A multilevel analysis of neighbourhood built and social environments and adult self-reported physical activity and body mass index in Ottawa, Canada. Environ Res Public Health 2011; 8:3953-78.

42. Wen M, Maloney TN. Latino residential isolation and the risk of obesity in Utah: the role of neighborhood socioeconomic, built-Environmental, and subcultural context. J Immigr Minor Health 2011; 13:1134-41.

43. Christian H, Giles-Corti B, Knuiman M, Timperio A, Foster S. The influence of the built environment, social environment and health behaviors on body mass index. Results from RESIDE. Prev Med 2011; 53:57-60.

44. Amesty SC. Barriers to physical activity in the Hispanic community. J Public Health Policy 2003; 24:41-58.

45. Jesus GM, Jesus EFA. Nível de atividade física e barreiras percebidas para a prática de atividade física entre policiais militares. Rev Bras Ciênc Esporte 2012; 34:433-48.

46. Cohen DA, Hunter G, Williamson S, Dubowitz T. Are food deserts also play deserts? J Urban Health 2016; 93:235-43.

47. Hollands S, Campbell MK, Gilliland J, Sarma S. Association between neighbourhood fast-food and full-service restaurant density and body mass index: a cross-sectional study of Canadian adults. Can J Public Health 2014; 105:172-8.
48. Hino AAF, Reis RS, Sarmiento OL, Parra DC, Brownson RC. The built environment and recreational physical activity among adults in $\mathrm{Cu}-$ ritiba, Brazil. Prev Med 2011; 52:419-22.

49. Augusto P, Rodrigues F, Melo MP, Assis MR, Palma A. Condições socioeconômicas e prática de atividades físicas em adultos e idosos: uma revisão sistemática. Rev Bras Ativ Fís Saúde 2017; 22:217-32.

50. Almeida LFF. Ambiente alimentar urbano de Viçosa (MG) e sua relação com o consumo de frutas e hortaliças por idosos: estudo de base populacional [Dissertação de Mestrado]. Viçosa: Universidade Federal de Viçosa; 2017.

51. Hemphill E, Raine K, Spence JC, SmoyerTomic KE. Exploring obesogenic food environments in Edmonton, Canada: the association between socioeconomic factors and fastfood outlet access. Am J Health Promot 2008; 22:426-32.

52. Le H, Engler-Stringer R, Muhajarine N. Walkable home neighbourhood food environment and children's overweight and obesity: proximity, density or price? Can J Public Health 2016;107:42.

53. Mendes LL, Nogueira H, Padez C, Ferrão M, Velásquez-Meléndez G. Individual and environmental factors associated for overweight in urban population of Brazil. BMC Public Health 2013; 13:988-94.

54. Cooksey-Stowers K, Chwartz MB, Brownell KD. Food swamps predict obesity rates better than food deserts in the United States. Int J Environ Res Public Health 2017; 14:E1366.

55. Lovasi GS, Hutson MA, Guerra M, Neckerman KM. Built environments and obesity in disadvantaged populations. Epidemiol Rev 2009; 31:7-20. 


\section{Abstract}

The aim of this study was to estimate associations between environmental variables and obesity in the adult population in a medium-sized Brazilian city. This was a cross-sectional study with individual data from a population-based study and environmental data obtained by direct observation, later georeferenced by the addresses. The sample included 965 adults 20 to 59 years of age, and the study used anthropometric, socioeconomic, demographic, behavioral, and self-rated health data. The outcome variable was obesity, defined as body mass index $(B M I) \geq 30 \mathrm{~kg} / \mathrm{m}^{2}$. Retail food outlets were classified as: supermarkets and establishments with a predominance of healthy, unhealthy, and mixed foods. Settings for physical activity were categorized as public and private. Characterization of the social environment used the census tracts' per capita income in tertiles and the crime rate. Binary logistic regression analysis was performed according to the generalized estimating equations model. An inverse association was found between density of public and private locations for physical activity and obesity $(\mathrm{OR}=0.95,95 \% \mathrm{CI}$ : 0.92-0.99; OR = 0.98, 95\%CI: 0.97-0.99) in models adjusted by individual and environmental variables. In all the models, the highest per capita income tertile was inversely associated with obesity $(p \leq 0.05)$. The food environment and crime rates were not independently associated with obesity. These findings suggest that the census tract's income and its environment for physical activity can orient public policies to decrease obesity in this city.

Obesity; Environment and Public Health; Adult Health

\section{Resumen}

El objetivo de este estudio fue estimar las asociaciones entre las variables ambientales y la obesidad en una población adulta de un municipio brasileño de tamaño medio. Se trata de un estudio transversal, con datos individuales de un estudio de base poblacional, $y$ datos ambientales obtenidos mediante la observación directa, posteriormente georreferenciados a partir de las direcciones. La muestra incluyó a 965 adultos de 20 a 59 años. Se utilizaron datos antropométricos, socioeconómicos, demográficos, comportamentales y de percepción de salud. La variable desenlace fue la obesidad, definida por indice de masa corporal (IMC) $\geq 30 \mathrm{~kg} / \mathrm{m}^{2}$. Los establecimientos de venta de alimentos se clasificaron en: supermercados, establecimientos con predominio de alimentos saludables, no saludables y mixtos. El ambiente para la práctica de actividad física se categorizó en público y privado. Para la caracterización del ambiente social se utilizó la renta per capita del censo en terciles y la tasa de criminalidad. Se realizó un análisis de regresión logística binaria por el modelo de ecuaciones de estimación generalizadas. Se verificó la asociación inversa entre la densidad de los lugares públicos y privados para la práctica de actividad física y obesidad (OR = 0,95, IC95\%: 0,92-0,99; OR = 0,98, IC95\%: 0,97-0,99), en modelos ajustados por variables individuales y ambientales. En todos los modelos el tercio de renta per capita más alto se asoció inversamente a la obesidad $(p \leq 0,05)$. El ambiente alimentario y las tasas de criminalidad no estuvieron asociados independientemente a la obesidad. Tales resultados sugieren que la renta procedente del censo y el ambiente de actividad física pueden dirigir politicas públicas, con el fin de disminuir la obesidad en el municipio.

Obesidad; Medio Ambiente y Salud Pública; Salud del Adulto
Recebido em 19/Jun/2018

Versão final reapresentada em 27/Dez/2018 Aprovado em 24/Jan/2019 